\title{
Search for Single Scattering Events
}

\author{
R. Ramachandran ${ }^{1}$, A. A. Deshpande ${ }^{2}$, B. W. Stappers ${ }^{3}$ \\ 1 NFRA, Postbus 2, 7990 AA Dwingeloo, The Netherlands \\ 2 Raman Rescarch Institute, Bangalore 560080, India \\ 3 Sterren. Inst., Kruislaan 403, 1098 SJ Amsterdam, The Netherlands
}

\section{Single/discrete Scattering Events}

Radio signals, during their passage through the intervening medium, are scattered due to irregularities in the density of free electrons in the interstellar medium. Signals from distant sources undergo, most often, strong 8 multiple scattering while the signals from nearby sources may be only weakly scattered even at meter wavelengths. It is likely that the scattering of signals from some nearby sources is possibly non-multiple in nature and hence may show a distinct signature of single or discrete scaltering events. In such a situation we receive, along with the direct unscattered signal, only a few discrete delayed versions of the signal. In such a case, it appears possible to probe the properties (such as the size and the density contrast) of the discrete density-irregularities responsible for the scattering, if the associated delays can be measured.

\section{Details of Observations and Analysis}

We observed PSR B0950+08 with the aim of looking for such single/discrete scattering events. Observations were made at $382 \mathrm{MHz}$ with a bandwidth of $10 \mathrm{MHz}$, with PuMa (Pulsar Machine) at Westerbork Synthesis Radio Telescope. The signal voltages were Nyquist sampled and recorded at $20 \mathrm{MHz}$ in both $\mathrm{X}$ and Y polarization channels. During the off-line processing, the on-pulse regions were selected and coherent dedispersion was performed on these "gated" voltage series. The procedure adopted to look for the expected signature of the possible discrete scattering events is as follows.

We looked for significant discrete features in the autocorrelation (AC) function computed from the voltage series. Since the expected values of the delays are of the order of a microsecond, there was no need to compute the AC function over a long delay range. The procedure consisted of computation of the power spectrum at each short interval of $102.4 \mu \mathrm{sec}$ (using $2^{11}$-point Fourier transforms) and inverse Fourier transforms of suitably corrected the power spectrum to obtain estimates of the $\mathrm{AC}$ function.

One of the important reasons for restricting each section length to 102.4 $\mu$ sec is that this pulsar shows significant microstructure with typical time scale of about $\sim 175 \mu \mathrm{sec}$. Any time length we consider for our analysis should therefore be less than this time scale, so that the contribution of the microstructure to the AC function can be reduced significantly, without affecting the signatures we are interested in. It should be emphasized that we analyze the spectrum 


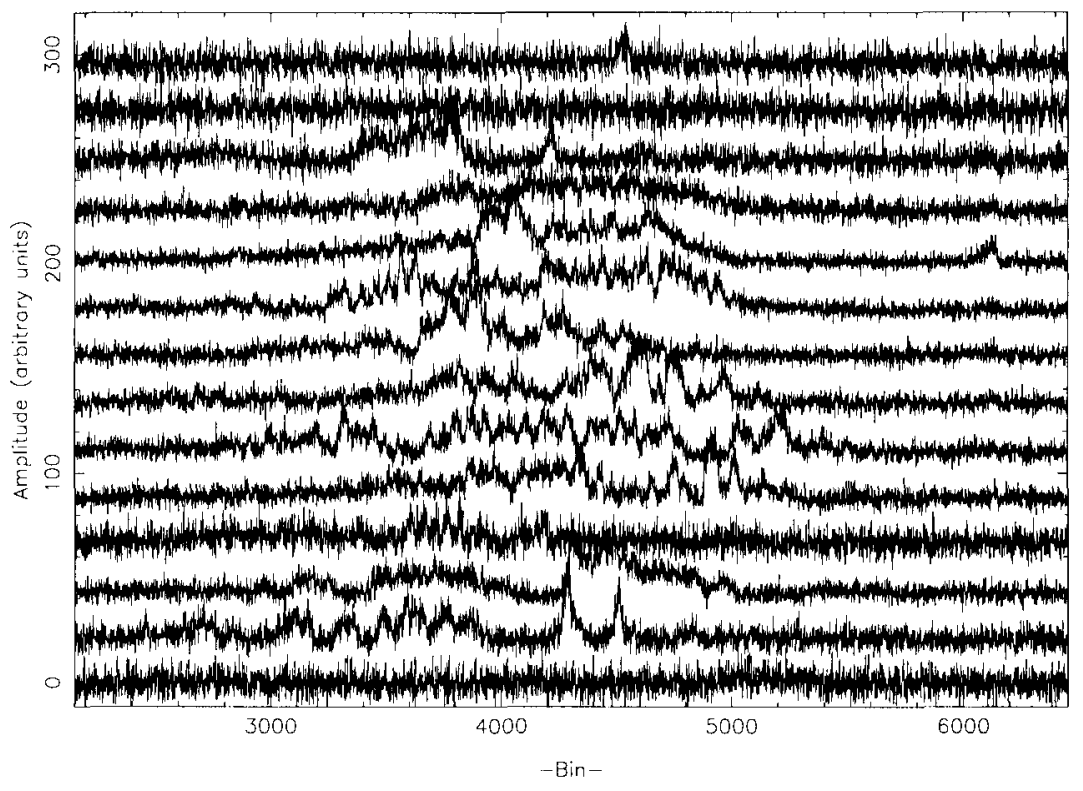

Figure 1. Single pulses from PSR B0950+08 after gating and coherent de-dispersion (and smoothing in time to $3.2 \mu \mathrm{sec}$ resolution). The $\mathrm{X}$-axis range corresponds to $\sim 30$ milliseconds.

of the voltage series as against the spectrum of the "detected" (i.e. the intensity) series. It is easy to see that the latter would correspond to a fluctuation spectrum of the intensity rather than the estimate of the radio-frequency spectrum we wish to study. Use of our procedure not only reduces the effect of any intrinsic fluctuations (such as the microstructure) in the signal strength, but allows us to measure the observable of interest more directly. Within the pulse window, AC functions were computed by taking half-overlapping time-sections of $2^{11}$ points. To improve the signal-to-noise ratio, all the individual $\mathrm{AC}$ functions were later added together with weights given by the pulse strength in the corresponding longitude span (which is estimated from the mean power in the spectrum). Furthermore, we repeat this procedure over many pulses and average all the individual $\mathrm{AC}$ functions, to compute the final average $\mathrm{AC}$ function.

Before looking for significant features in the AC function, it is important to estimate and correct for all the instrumental effects. There are two major effects to be considered: (1) intrinsic bandshape of the instrument, and (2) the telescope front-end response. The reason for considering the front-end (the signal path before the first amplifier) as a separate entity is that a very significant fraction of the system noise is contributed by the first amplifier which does not sample the spectral response of the front-end. This means that the spectral response estimation based on off-pulse signal (dominated by the receiver noise power) cannot be used, in general, to adequately correct for the instrumental spectral response to the sky/pulsar signal. 


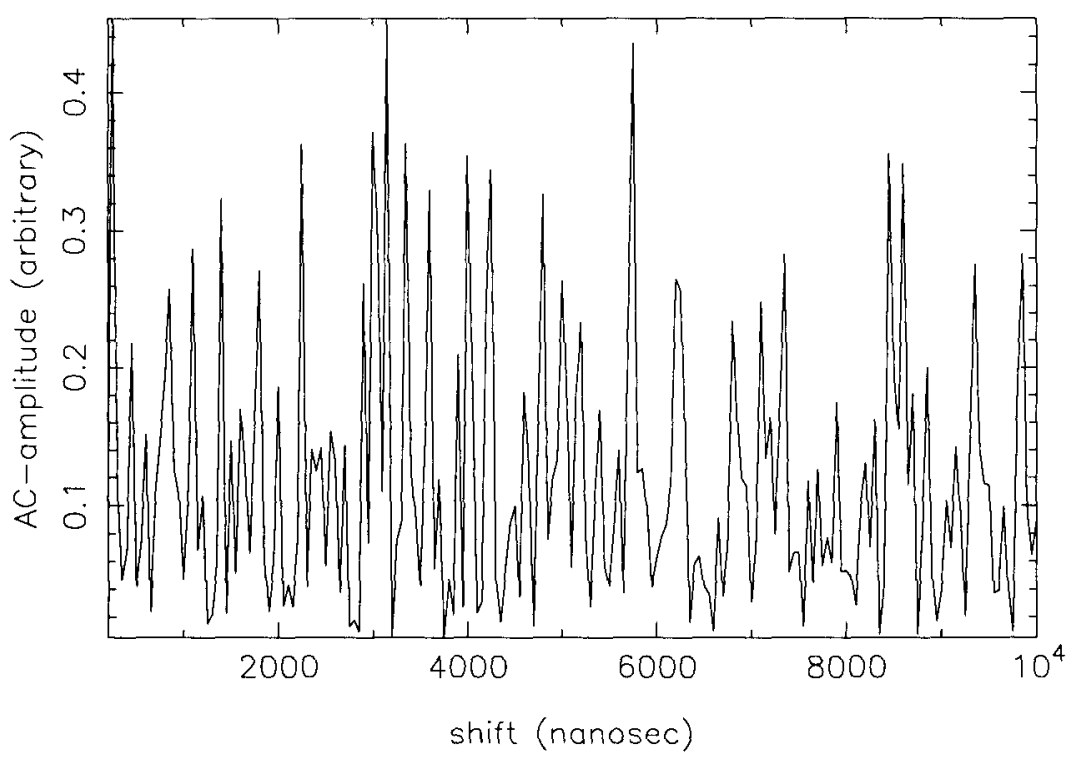

Figure 2. Autocorrelation function averaged over 120 pulses. See text for details.

We therefore model the instrumental-response function as follows:

$$
B_{\text {on }}(\nu)=\left\langle B_{\text {off }}\right\rangle(\nu)\left[1+\left(\frac{A_{\text {on }}-\left\langle A_{\text {off }}\right\rangle}{\left\langle A_{\text {off }}\right\rangle}\right) D(\nu)\right]
$$

Here, $B_{\text {on }}(\nu)$ is the on-pulse bandshape as a function of frequency (obtained for a given stretch of $2^{11}$ points), $\left\langle B_{\text {off }}\right\rangle(\nu)$ is the average off-pulse bandshape as a function of frequency, $A_{\text {on }}$ is the mean of the on-pulse instantaneous bandshape, and $\left\langle A_{\text {off }}\right\rangle$ is the mean of the average off-pulse bandshape. $D(\nu)$ is the front-end function. While computing the AC function, we corrected for these instrumental effects in the band, so that we have an unbiased $\mathrm{AC}$ function. Also the mean spectral power was estimated for each of the on-pulse spectra and removed from the spectrum before Fourier transforming to obtain a (residual) AC function.

A sample section of the pulse sequence used for our analysis is shown in Figure 1. The final autocorrelation, function after averaging over 120 bright pulses is given in Figure 2. As we can see, there is no statistically significant feature in the autocorrelation function, indicating the absence of any significant single/discrete scattering event.

For a ray passing through a spherical cloud/irregularity of radius $a$, with an associated electron density fluctuation $\delta n_{e}$, the extra phase introduced for a wave of frequency $\nu$ travelling through this cloud is $\delta \phi=2 a e^{2} \delta n_{e}(m c \nu)^{-1}$, where $m \& e$ are the mass and the charge of an electron, respectively, and $c$ is the velocity of light in vacuum. Expressing the time delay between the "direct" ray and the scattered ray, we get 


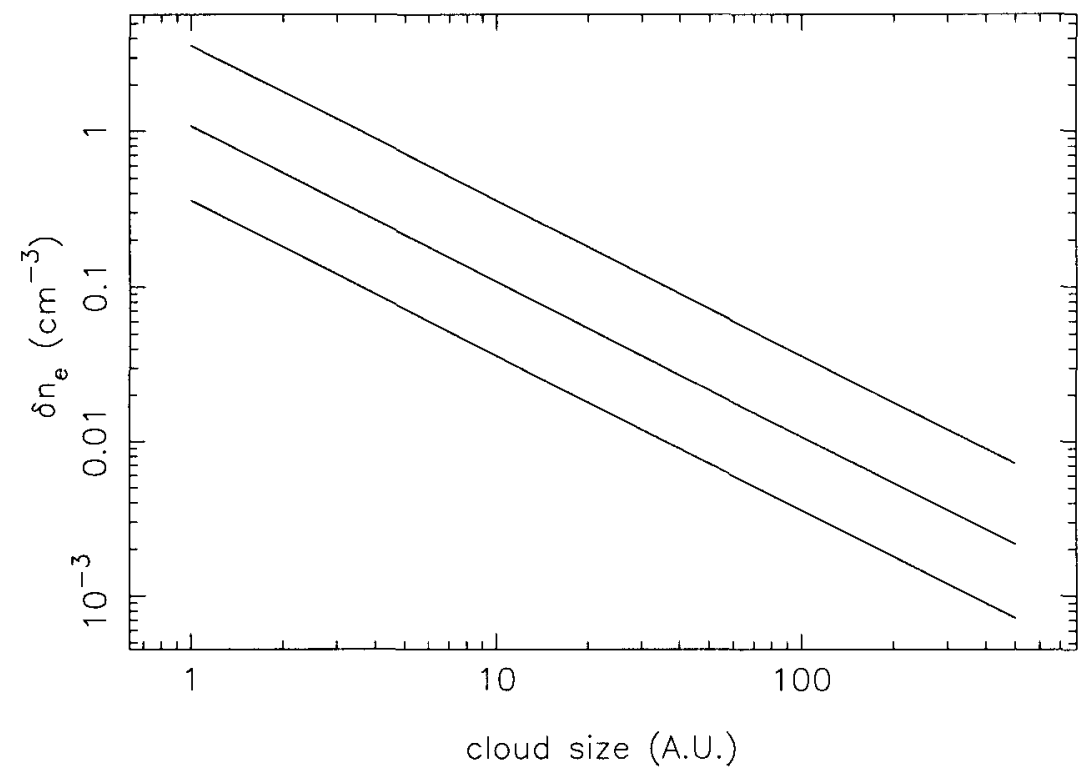

Figure 3. Cloud size vs $\delta n_{e}$ for various assumed time delays for feature autocorrelation function. The three curves (from top to bottom) are for the delay values of 1000,300 and 100 nanoseconds.

$$
\tau_{\mathrm{sc}}=\left(\frac{d e^{4} \delta n_{e}^{2} \lambda^{4}}{4 \pi^{2} c^{5} m^{2}}\right)^{1 / 2}
$$

Substituting in the RHS of this equation $\tau_{\mathrm{sc}}=(4 a / c d)$, we get the relation

$$
\delta n_{e}=\left(\frac{\pi c^{3} m \tau_{\mathrm{sc}}}{e^{2} \lambda^{2} a}\right)
$$

For a feature corresponding to a certain delay value in the $\mathrm{AC}$ function, Equation 3 gives a relation between the number density contrast and the size of the irregularity. Figure 3 shows this relation for various assumed time delay $\left(\tau_{\mathrm{sc}}\right)$ values. The three curves (from top to bottom) correspond to delay values of 1000,300 and 100 nanoseconds. If we assume that the minimum measurable delay in our observations is 100 nanosec (reciprocal of $10 \mathrm{MHz}$ ), then the bottom most curve gives the relation between the irregularity/cloud size and the maximum value of the associated $\delta n_{e}$ of the cloud. Therefore, this is the upper limit to the $\delta n_{e}$ derived by our observations.

Results of our analysis of observations over a few different epochs of this pulsar (B0950+08) and PSR B1929+10 will be presented elsewhere (Ramachandran, Deshpande \& Stappers 2000). 